\title{
Live Demonstration: Low-Power Low-Cost Cyber-Physical System for Bird Monitoring
}

\author{
A. García-Rodríguez*, R. Rodríguez-Sakamoto*, J. Fernández-Berni*, R. del Río*, J. Marín ${ }^{\dagger}$, M. Baena ${ }^{\dagger}$, \\ J. Bustamante ${ }^{\dagger}$, R. Carmona-Galán* and Á. Rodríguez-Vázquez* \\ *Instituto de Microelectrónica de Sevilla (Universidad de Sevilla-CSIC), 41092 Seville, Spain \\ †Estación Biológica de Doñana (EBD-CSIC), 41092 Seville, Spain
}

\begin{abstract}
This live demonstration showcases a cyber-physical system tailored for inexpensive remote bird monitoring. A comprehensive analysis of the application requirements along with a tight system integration have given rise to a smart autonomous nest-box ready for deployment. This nest-box includes radiofrequency identification (RFID), a weighing scale, two temperature sensors, passive infrared devices (PIR), massive data storage and internet connection via mobile infrastructure. It is powered through a solar panel. The bill of materials has been diminished $77 \%$ with respect to the previous version of the nest-box whereas the power consumption has been reduced $84 \%$.
\end{abstract}

\section{INTRODUCTION}

Cyber-physical systems - i.e. smart systems that include engineered interacting networks of physical and computational components [1] - are the primary constituent elements of the Internet of Things (IoT). A major requirement for a wide adoption of the latter is ultra-low-power low-cost realizations of the former [2]. This would also enable the practical operation of applications demanding massive deployments, e.g. nature monitoring. Indeed, IoT devices are expected to play a crucial role in nature conservation [3]. This demo fits this particular application scenario. We showcase the next generation of a smart nest-box for automatic bird monitoring [4]. The main requirements for the evolved implementation of this cyber-physical system were: i) reduction of power consumption aiming at autonomous operation from a solar panel; ii) reduction of bill of materials targeting low-cost field deployment; iii) wireless communication; and iv) better integration of sensing capabilities for progressive activation of the various components according to bird behavior. Keeping Arduino as the base platform, we have designed a system meeting these requirements. Its power consumption has been reduced from $18.58 \mathrm{~W}$ to $2.9 \mathrm{~W}$. A standby mode has been incorporated featuring a consumption of $500 \mathrm{~mW}$. The bill of materials has diminished from over $2000 €$ to roughly $500 €$.

\section{LiVE Demonstration SETUP}

The nest-box is shown in Fig. 1. Since the demo will take place indoors, Arduino will be powered through a pre-charged LiPo battery. In real operating conditions, this battery would be recharged by the solar panel. The control flow performed by Arduino encompasses two stages. On booting, the system parameters are initialized and the sensors are calibrated. An infinite loop is then executed, waiting for meaningful sensing

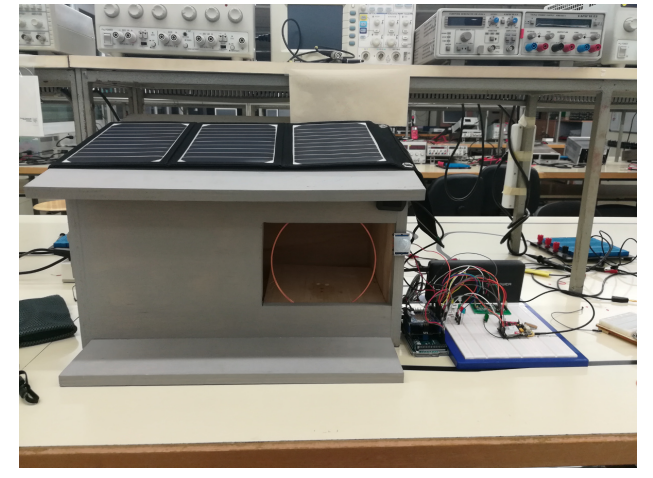

Fig. 1. Arduino-based cyber-physical system for bird monitoring.

events. The activation of a particular PIR sensor correspondingly triggers the RFID module or the weighing scale. We have also encoded the activation of a camera to be integrated in the near future. Each sensing event is time-stamped and stored in an SD card. If needed, these events can be transmitted via a GSM/GPRS module.

\section{VISITOR EXPERIENCE}

The visitors will be able to test the different sensing capabilities of the system. Communication with the nest through any mobile phone will also be possible.

\section{ACKNOWLEDGMENT}

This work has been funded by the Spanish Government, project TEC2015-66878-C3-1 MINECO (European Region Development Fund, ERDF/FEDER) and by Junta de Andalucía, project TIC 2338-2013 CEICE. Appreciation to Mr. A. García-Cháves, Mr. E. Martín-Sotelo and Mr. P. SánchezFernández for their help to set up the system.

\section{REFERENCES}

[1] Framework for Cyber-Physical Systems, National Institute of Standards and Technology, United States.

[2] M. Alioto, E. Sánchez-Sinencio, and A. Sangiovanni-Vincentelli, "Guest editorial: Special issue on circuits and systems for the internet of thingsfrom sensing to sensemaking," IEEE Trans. Circuits Syst. I, vol. 64, no. 9, pp. 2221-2225, 2017.

[3] WILDLABS, The Conservation Technology Network. [Online]. Available: https://www.wildlabs.net/ (accessed on November 15, 2017)

[4] D. Larios et al., "An automatic weighting system for wild animals based in an artificial neural network: How to weigh wild animals without causing stress," Sensors, vol. 13, pp. 2862-2883, 2013. 